\title{
Pharmacists and medication adherence in asthma: a systematic review and meta-analysis
}

\author{
Marissa Ayano Mes ${ }^{1}$, Caroline Brigitte Katzer ${ }^{1}$, Amy Hai Yan Chan ${ }^{1}$, \\ Vari Wileman ${ }^{1}$, Stephanie Jane Caroline Taylor ${ }^{2}$ and Rob Horne ${ }^{1}$
}

Affiliations: ${ }^{1}$ Centre for Behavioural Medicine, Dept of Practice and Policy, UCL School of Pharmacy, University College London, London, UK. ${ }^{2}$ Centre for Primary Care and Public Health, Blizard Institute, Queen Mary University of London, London, UK.

Correspondence: Rob Horne, Centre for Behavioural Medicine, UCL School of Pharmacy, Mezzanine Floor, BMA House, Tavistock Square, London WC1H 9JP, UK. E-mail: r.horneducl.ac.uk

@ERSpublications

Pharmacist-led interventions can improve medication adherence in people with asthma http://ow.ly/IefK30kA6Z5

Cite this article as: Mes MA, Katzer CB, Chan AHY, et al. Pharmacists and medication adherence in asthma: a systematic review and meta-analysis. Eur Respir J 2018; 52: 1800485 [https://doi.org/10.1183/ 13993003.00485-2018].

ABSTRACT The Lancet Asthma Commission highlighted that non-adherence remains a persistent barrier within asthma care. Medical consultations remain pivotal in addressing non-adherence, but interest in additional adherence support from pharmacists is increasing. This systematic review and meta-analysis aims to evaluate how effective pharmacist-led interventions are in improving medication adherence in adults with asthma.

Studies were included if they had adult participants with asthma, pharmacist-led or collaborative care interventions, randomised controlled trial designs comparing interventions with usual pharmacist care, and a medication adherence outcome. We analysed adherence data using the standardised mean difference $(d)$ and the remaining data were synthesised narratively.

From 1159 records, 11 were included in the narrative synthesis and nine in the meta-analysis. The meta-analysis for adherence produced a medium effect size of $d=0.49$ ( $\mathrm{sE}=0.08,95 \%$ CI $0.35-0.64$, $\mathrm{p}<0.0001)$ with low statistical heterogeneity.

In line with the Perceptions and Practicalities Approach, interventions targeted to address both the perceptions and practicalities that influence individual motivation and ability to adhere were more effective. Contextual factors (country and healthcare setting) were also influential. Our findings suggest that with adequate remuneration and integration into asthma care, pharmacists can help improve adherence in asthma. 


\section{Introduction}

There are over 300 million people with asthma worldwide and this figure is set to increase by 100 million by 2025 [1,2]. Research has consistently demonstrated a link between asthma, poor health outcomes and substantial healthcare costs [3-6]. Better implementation of current diagnosis and management strategies may help reduce the impact of asthma globally. However, global asthma mortality rates have not changed in over a decade and novel approaches to support people with asthma should also be considered [3, 7].

Adherence to inhaled preventive asthma medication is suboptimal in $>50 \%$ of people with asthma, with the recent Lancet Asthma Commission calling for more effective methods of identifying and addressing non-adherence $[7,8]$. Although the medical consultation is pivotal, there is increasing interest in additional support delivered outside the consultation. Due to the growing pressure on primary care practitioners, interest in pharmacist-led adherence support has grown, as demonstrated by recent healthcare initiatives and government policy [9-12]. Although several studies have examined pharmacist-led adherence support, this literature has not been reviewed.

The aims of our systematic review and meta-analysis were to determine 1) the overall effectiveness of pharmacist-led adherence support for adults with asthma; 2) whether the content of interventions influenced their effectiveness, in particular, whether or not they applied adherence theory and behaviour change strategies; and 3) whether the context of interventions (country and healthcare setting) influenced their effectiveness [13].

\section{Methods}

We conducted a systematic review and meta-analysis. Our protocol was registered on the International Prospective Register of Systematic Reviews (record CRD42016035657, www.crd.york.ac.uk/PROSPERO) by the Centre for Reviews and Dissemination. The review is reported in accordance with the Preferred Reporting Items for Systematic Reviews and Meta-Analyses (PRISMA) guidelines [14].

\section{Search strategy}

We searched four pharmacy-relevant databases (Embase, MEDLINE, Web of Science, and CENTRAL). The search began on May 5, 2016, with weekly alerts for new publications until the last search on June 15, 2017. Our search strategy used database-specific vocabulary (e.g. Medical Subject Headings) and free text expanding from "pharmacist" and "asthma". Because we wanted to capture studies even if adherence was not a primary intervention target, "adherence" was not included as a search term. Cochrane Collaboration search filters for identifying randomised controlled trials (RCTs) were used on MEDLINE (sensitivity-maximising) and Embase (sensitivity-and-precision maximising) [15, 16]. We included literature published in English, Spanish, German and Dutch (languages spoken by the research team). There were no restrictions on publication date.

\section{Study selection}

EndNote Reference Manager (Clarivate Analytics, Philadelphia, PA, USA) was used to store records and remove duplicates. Authors of studies were contacted if further information was needed for an inclusion decision. One investigator (M.M.) screened all titles and abstracts. Inclusion decisions were cross-checked with other research team members (C.K., R.H. and S.T.). Two investigators (C.K. and M.M.) independently screened full-text articles. All inconsistent inclusion decisions were resolved through consensus.

Inclusion criteria followed the PICOS (participant, intervention, comparison, outcome, study design) framework [15]. Participants were adults with asthma and no other respiratory conditions, with a prescription for asthma medication. There were no restrictions on how asthma was diagnosed. We excluded participants with other respiratory conditions because their medication and adherence behaviour may be different to that of participants with only an asthma diagnosis. During our initial searches, we found many studies that included underage $(\leqslant 17$ years) and adult $(\geqslant 18$ years) participants. Separate group data were often unavailable. We therefore included studies if the majority of participants were adults.

Intervention content could be partially or completely delivered by a pharmacist (i.e. pharmacist-led). Collaborative care interventions delivered with other healthcare professionals (e.g. nurses) were included only if pharmacists were involved in delivering intervention content. We excluded studies in which pharmacists only assisted in research activities (e.g. recruitment). Studies had to compare pharmacist-led interventions with usual pharmacist care in an RCT design. Usual pharmacist care was defined as dispensing and providing basic information about asthma and its treatment.

We included studies with adherence as a primary or secondary outcome. We focused on summary estimates, rather than participant-level data. Where studies used multiple measures of adherence, the most objective measure was included in the following order: electronic adherence monitoring devices 
(e.g. Doser ${ }^{\mathrm{TM}}$, Smartinhaler ${ }^{\mathrm{TM}}$ ), pharmacy-based data (e.g. prescription refill rates), patient self-report measures, and reports from healthcare professionals or carers. RCTs were randomised at the participant or cluster level. We focused only on RCTs to compare data of a similar methodological standard.

\section{Data collection}

Authors of included studies were contacted for further information about study design and intervention content. Published protocols were consulted where possible. We used the Cochrane Collaboration's tool to assess risk of bias [17]. We extracted data about research design, location, healthcare setting, participants, outcome measures, intervention delivery and intervention content (supplementary table S1). One investigator (M.M.) extracted these data across all studies. Another investigator (C.K.) independently extracted intervention content data and coded risk of bias across $25 \%$ of included studies for cross-checking.

We categorised intervention content based on 1) whether it was informed by a Perceptions and Practicalities Approach (PAPA), as recommended by the National Institute for Health and Care Excellence (NICE) guidelines for medicines adherence (figure 1) $[18,19]$; and 2) the implemented Behaviour Change Techniques (BCTs) as defined by Michie et al. [20]. According to the PAPA, tailored interventions targeted to address the perceptions (e.g. beliefs about illness and treatment) and practicalities (e.g. capability and resources) affecting motivation and ability to adhere to treatment are more effective [18].

Interventions were categorised as non-PAPA (targeting only perceptions or practicalities), partial PAPA (targeting perceptions and practicalities) or full PAPA interventions (tailored approach targeting perceptions and practicalities). The partial PAPA category can also include interventions which address either perceptions or practicalities in a tailored manner. However, for this review, partial PAPA interventions were defined only as those addressing perceptions and practicalities, because the level of detail provided in the studies about intervention tailoring was insufficient for coding. The BCT Taxonomy is a classification that outlines standardised techniques that can change behaviour when applied in the right context (e.g. "demonstration of a behaviour" to teach inhaler technique) [20]. It is a useful tool to identify exactly which parts of an intervention are effective.

\section{Data synthesis}

For our narrative synthesis, we compared studies that found an intervention effect (positive studies) with those that did not (negative studies). We first identified differences based on risk of bias, research design, location, healthcare setting, participants, outcome measures, and intervention delivery to see if they affected adherence outcomes. We then compared studies based on intervention content to see if specific components of interventions improved adherence.

Where feasible and appropriate, we conducted a meta-analysis using the standardised mean difference $(d)$. We chose a random effects model due to the expected heterogeneity of participants and interventions. We chose $d$ because we assumed that adherence is a continuous construct regardless of how it is measured. Furthermore, data had to be standardised for comparison because studies used different adherence measures [15]. Where necessary, we adjusted cluster-randomised studies by inflating standard errors using an intra-cluster correlation coefficient of 0.05 , based on previous estimates [21, 22]. We assessed

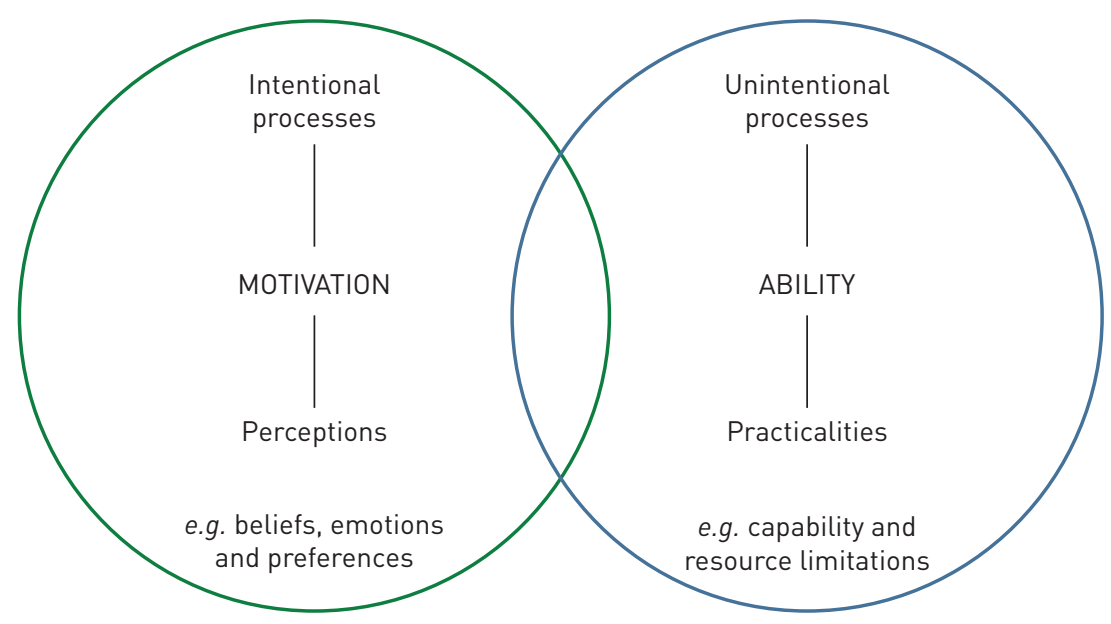

FIGURE 1 The Perceptions and Practicalities Approach. 
heterogeneity (Chi-squared test, $I^{2}$ statistic, forest plots) and publication bias (funnel plots, fail-safe N). We conducted sensitivity analyses for the intra-cluster correlation coefficient used $(0.01,0.05,0.07$, or 0.10 ), risk of bias (high-risk studies removed), mixed age samples (studies with participants $<18$ years removed) and meta-analysis model (fixed and random effects).

\section{Results}

\section{Study characteristics}

A total of 843 studies were retrieved from the databases. We excluded 797 studies based on titles and abstracts and 35 studies were excluded based on full-text articles (figure 2). Primary reasons for exclusion were: same participant sample, not asthma patients, not RCT, author non-response to request for information, no adherence measure, not pharmacist-led, no usual care control group and unpublished studies (figure 2). We included 11 studies in the narrative synthesis [23-33] and nine studies in the meta-analysis [23-26, 28, 30-33]. Authors of eight included studies provided further information upon request $[23-25,28,29,31-33]$. Two authors did not respond [26, 30]. One author was unable to provide further details due to intellectual property restrictions [27].

Five studies $(45 \%)$ were cluster-randomised by pharmacy [23, 25-27, 31]. The remaining studies were randomised at the participant level (table 1). All studies met the criteria for a usual pharmacist care control group. However, three of these studies (27\%) were delivered through clinical settings (hospitals and federal health clinics) with a possibly better quality of usual care [30, 32, 33]. One study (9\%) gave control participants an educational asthma booklet [24] and one study (9\%) provided inhaler technique training [32]. Studies had a follow-up period of four [32], six [23, 24, 26, 28, 30, 31, 33], 5.3 [29], nine [27] and

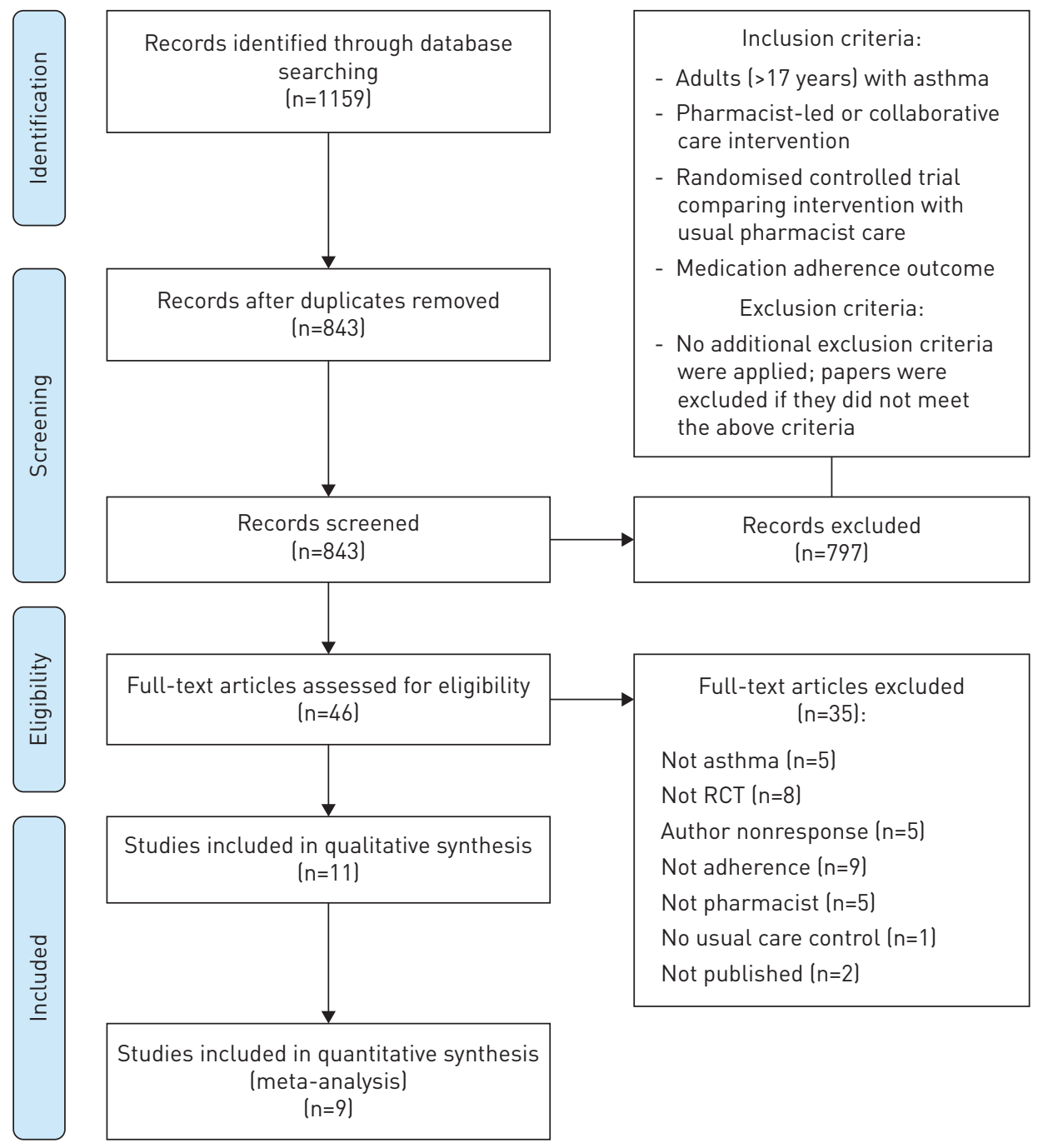

FIGURE 2 PRISMA (Preferred Reporting Items for Systematic Reviews and Meta-Analyses) flow diagram for study inclusion. RCT: randomised controlled trial. 
TABLE 1 Research design, location and outcome characteristics of included studies

\begin{tabular}{|c|c|c|c|c|c|c|c|c|}
\hline Study & Randomised & $\begin{array}{l}\text { Intervention } \\
\text { group } \mathrm{n}\end{array}$ & $\begin{array}{l}\text { Control } \\
\text { group n }\end{array}$ & $\begin{array}{l}\text { Follow-up } \\
\text { months }\end{array}$ & Country & $\begin{array}{l}\text { Healthcare } \\
\text { setting }\end{array}$ & Adherence measure & $\begin{array}{l}\text { Significant } \\
\text { intervention } \\
\text { effect? }\end{array}$ \\
\hline ChARRoIs et al., 2006 [24] & Patient & 36 & 34 & 6 & Canada & Community & $\begin{array}{l}\text { Prescription refill } \\
\text { rates }\end{array}$ & No \\
\hline CoRdina et al., 2001 [25] & Cluster & 64 & 55 & 12 & Malta & Community & $\begin{array}{l}\text { Self-reported rates of } \\
\text { forgetting per day }\end{array}$ & No \\
\hline MANFrIN et al., 2017 [27] & Cluster & 400 & 416 & 9 & Italy & Community & $\begin{array}{l}2 \text { items from } \\
\text { MMAS- } 8\end{array}$ & Yes \\
\hline Menurs et al., 2008 [28] & Patient & 80 & 70 & 6 & Belgium & Community & $\begin{array}{l}\text { Prescription refill } \\
\text { and self-report: } \\
\text { "How often do you } \\
\text { not take your } \\
\text { controller as } \\
\text { prescribed?" }\end{array}$ & Yes \\
\hline WANG et al., 2010 [30] & Patient & 29 & 32 & 6 & Taiwan & Outpatient & MMAS-4 & No \\
\hline Wong et al., 2017 [31] & Cluster & 80 & 77 & 6 & Malaysia & $\begin{array}{l}\text { Outpatient and } \\
\text { telepharmacy }\end{array}$ & MALMAS & Yes \\
\hline $\begin{array}{l}\text { XAUBet OLivera et al., } \\
2016 \text { [32] }\end{array}$ & Patient & 52 & 53 & 4 & Brazil & Outpatient & $\begin{array}{l}\text { MMAS }-4 \text { and } \\
\text { prescription refill } \\
\text { rates }\end{array}$ & Yes \\
\hline Young et al., 2012 [33] & Patient & 41 & 42 & 6 & USA & Telepharmacy & MMAS-8 & No \\
\hline
\end{tabular}

MMAS: Morisky Medication Adherence Scale (4 and 8 items); MALMAS: Malaysian Medication Adherence Scale. ${ }^{\#}$ : community indicates community pharmacy, outpatient indicates ambulatory care/outpatient clinics, telepharmacy indicates telephone-based; १: yes indicates a significant intervention effect $(p<0.05)$ and no indicates no significant intervention effect $(p \geqslant 0.05)$.

12 months [25]. Studies were conducted in Australia [23], Canada [24], Malta [25], Spain [26], Italy [27], Belgium [28], Taiwan [30], Malaysia [31], Brazil [32] and the USA [29, 33]. Most interventions (64\%) were delivered in community pharmacies. Two interventions (18\%) were delivered in outpatient clinics [30, 32]. One intervention (9\%) was delivered completely over the telephone [33].

TABLE 2 Participant characteristics of included studies

\begin{tabular}{|c|c|c|c|c|c|}
\hline Study & Age years & Male sex & Uptake & Attrition & Asthma control \\
\hline ARMour et al., 2007 [23] & $50.2 \pm 16.4$ & 35.0 & & 11.4 & Mixed \\
\hline ChARRoIs et al., 2006 [24] & $37.2 \pm 10.5$ & 47.2 & 77.4 & 1.4 & Uncontrolled only \\
\hline CoRdina et al., 2001 [25] & $43.2 \pm 18.3$ & 50.6 & & 21.7 & Mixed \\
\hline García-CÁrdenas et al., 2013 [26] & $55.8 \pm 19.1$ & 46.1 & 97.1 & 9.9 & Mixed \\
\hline MANFRIN et al., 2017 [27] & $54.1 \pm 17.2$ & 41.2 & & 35.4 & Mixed \\
\hline Menuys et al., 2008 [28] & $35.7 \pm 5.5$ & 46.9 & 72.8 & 25.4 & Mixed \\
\hline MunZenberger and Hill 2007 [29] & $36.1 \pm 14.5$ & 23.3 & 100 & 26.8 & Uncontrolled only \\
\hline WANG et al., 2010 [30] & $28.2 \pm 10.9$ & 72.1 & & 11.6 & Mixed \\
\hline Wong et al., 2017 [31] & $55.2 \pm 13.3$ & 46.5 & 79.5 & 8.2 & Mixed \\
\hline X ХuUbet Olivera et al., 2016 [32] & $52.0 \pm 10.2$ & 27.6 & & 11.8 & Mixed \\
\hline Young et al., 2012 [33] & $44.6 \pm 15.8$ & 23.5 & 77.8 & 15.3 & Mixed \\
\hline Overall & $50.2 \pm 16.0$ & $41.8 \pm 14.2$ & $84.1 \pm 11.5$ & $16.3 \pm 9.9$ & \\
\hline
\end{tabular}

Data are presented as mean \pm SD or percentages, unless otherwise indicated. " : mixed asthma indicates mixed levels of controlled, partly controlled and uncontrolled asthma. 
Five studies (45\%) reported on adherence as a primary outcome [25, 30-33]. The most common outcome reported was asthma control (73\% of studies) [23-28, 31, 33]. Adherence was most commonly measured using validated self-report measures (55\%) [23, 26, 30-33], followed by non-validated self-report measures (36\%) $[25,27-29]$ and prescription refill data $(27 \%)[24,28,32]$ (table 1$)$.

\section{Participant characteristics}

The mean \pm SD age across the review was $50.2 \pm 16.0$ years (table 2 ). Four studies (36\%) included a small number of participants $<18$ years; the maximum percentage of participants $<18$ years in any one study was $23 \%[24,25,28,29]$. The mean \pm SD percentage of male participants was $41.8 \pm 14.2 \%$. The median sample size across studies was 119 , with an interquartile range (IQR) of 170. The median sample size was 64 (IQR 84) for the intervention groups and 55 (IQR 75.5) for the control groups. The mean \pm SD rate of study uptake (percentage of study invitations accepted per invitations extended) was high $(84.1 \pm 11.5 \%)$ for the six studies that reported this data $[24,26,28,29,31,33]$. The mean \pm SD percentage of participants lost to follow-up was $16.3 \pm 9.9 \%$.

Four studies $(36 \%)$ had equal proportions of uncontrolled, controlled and partly controlled asthma in their samples $[25,30,31,33]$. Three studies $(27 \%)$ had samples with a higher proportion of uncontrolled asthma [23, 26, 32]. Two studies (18\%) had a higher proportion of controlled and partly controlled asthma in their samples $[27,28]$. Two studies (18\%) focused only on uncontrolled asthma $[24,29]$.

\section{Risk of bias}

Studies were categorised as having a low [31, 32], moderate [23, 26, 28, 33] or high risk of bias [24, 25, 27, 29,30 ] (supplementary figure S1). The overall risk of bias across the review was moderate to high. There was a high risk of performance bias in all studies, partly because it is difficult to blind participants in behavioural intervention trials as the intervention and control conditions are noticeably different. Consequently, eight studies $(73 \%)$ were judged as having a high risk of detection bias because they relied on self-report measures of adherence with non-blinded participants [23, 25-27, 29-31, 33]. Other sources of bias included possible contamination between groups and use of non-validated measures of adherence [24, 25, 27-29, 33].

\section{Effects on medication adherence}

Six studies (55\%) found a significant intervention effect on medication adherence (positive studies) [23, $26-28,31,32]$. We adjusted estimates from three studies for clustering $[23,25,26]$. The random effects meta-analysis produced a medium effect size \pm SE of $d=0.49 \pm 0.08$ (95\% CI $0.35-0.64, \mathrm{p}<0.0001)$ [34]. There was low statistical heterogeneity in the analysis according to the Chi-squared statistic $(9.84, \mathrm{df}=8, \mathrm{p}=0.28)$, $I^{2}$ statistic (16.42\%) and forest plot (figure 3). Owing to the small number of studies in the meta-analysis, we were unable to conduct moderator and publication bias analyses. Results remained consistent across all sensitivity analyses.

\section{Intervention delivery}

With regards to intervention delivery, pharmacists were trained using self-study manuals and/or interactive workshops. Teaching methods included role play and feedback, patient actors, group discussions and question-and-answer sessions [23-25, 27, 29, 33]. Training was led by communication reviewers [24, 33], researchers $[23,25,29]$ and previously trained pharmacists [28]. Nine interventions $(81 \%)$ were delivered exclusively by pharmacists. Two interventions (18\%) were delivered by pharmacists in collaboration with nurses [30], or respiratory therapists and physicians [24]. One intervention (9\%) was delivered over the telephone in individual sessions [33]. Another intervention was delivered face-to-face in group sessions with other asthma patients [32]. The remaining interventions (81\%) were delivered face-to-face in individual sessions.

\section{Intervention content}

Five studies (45\%) were categorised as non-PAPA interventions because they focused solely on increasing people's knowledge about asthma through education, rather than addressing people's perceptions of asthma and/or asthma treatment [24, 25, 28-30]. Although limited knowledge of a chronic condition can lead to poor health outcomes, an educational approach only addresses the practicalities affecting adherence (i.e. knowledge) [35]. Research shows that increased knowledge does not necessarily guarantee behaviour change [36, 37]. One study (9\%) was categorised as a partial PAPA intervention because it targeted perceptions (e.g. beliefs about asthma and its treatment) as well as practicalities (e.g. inhaler technique). However, it was delivered in a group setting and this limited the pharmacist's ability to tailor adherence support [32]. The remaining five studies (45\%) were categorised as full PAPA interventions because they targeted perceptions and practicalities in a tailored manner [23, 26, 27, 31, 33]. In terms of how interventions tried to change adherence, we coded BCTs across eight categories: goals and planning, 


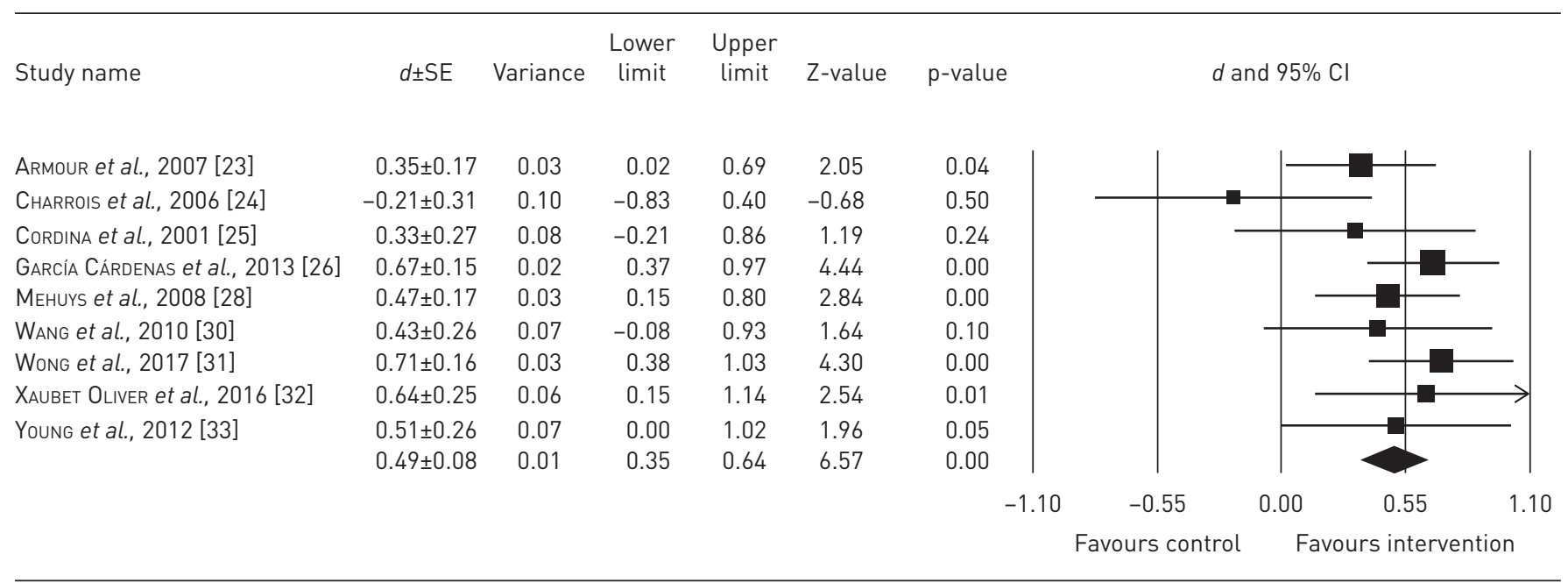

FIGURE 3 Forest plot for meta-analysis of medication adherence outcomes. $d$ : standardised mean difference.

feedback and monitoring, shaping knowledge, comparison of behaviour, repetition and substitution, natural consequences, self-belief, and associations (supplementary table S2).

\section{Discussion \\ Main findings}

This is the first systematic review and meta-analysis of pharmacist-led adherence support in adults with asthma. Our findings show that pharmacist-led interventions can significantly improve medication adherence (medium effect size in meta-analysis, $d=0.49$ ) [34]. In line with the NICE guidelines, pharmacist-led interventions applying a full PAPA were more effective $[18,19]$. The type and number of BCTs used in the intervention did not determine effectiveness, though this finding may be due to insufficient data to accurately code BCTs.

Intervention context (country and healthcare setting) may have influenced effectiveness. Interventions delivered in Australia, Belgium, Spain and Italy may have benefited from pharmacist involvement in public health policy [38], adequate pharmacist training and remuneration [27, 38-40] and/or more pharmacists per 100000 people [41]. In contrast, the healthcare systems in Canada, Malta, and the USA posed barriers such as fewer pharmacists per 100000 people [42], asthma only being treated in secondary care [25] and limited private health insurance coverage beyond medication dispensing [43].

Our findings are in line with a recent Cochrane review of interventions targeting adherence to inhaled corticosteroids. The review found a significant intervention effect on adherence when compared to usual care [44]. In terms of intervention location, previous research looking at pharmaceutical care activities and resources across Europe also found significant differences between countries [45].

\section{Limitations of included studies}

None of the studies were able to blind their participants to allocation and this may have significantly affected study outcomes [46]. Studies that used self-report measures of adherence with non-blinded participants were subject to detection bias. Self-report measures of adherence are limited when participants are untruthful [47]. Furthermore, participant behaviour in behavioural intervention trials can be affected by the act of filling in a questionnaire [48]. Research suggests that prescription refill data is a more reliable proxy measure of adherence [49]. However, it is based on the assumption that medication is taken correctly. This is problematic when it comes to asthma medication, where patients may be losing a proportion of each dose due to poor inhaler technique [50].

Contamination between the intervention and control groups may have been an issue in the studies randomised at the participant level. Few of the included studies examined intervention fidelity, with only two studies reporting measures to ensure the intervention was being delivered as intended [23, 26]. The quality of information available about intervention tailoring was insufficient for most studies and we were unable to use the alternative criteria for coding partial PAPA interventions. Most studies had a follow-up period of 6 months, making it difficult to assess the sustainability of intervention effects. 
Strengths and limitations of the current review

This review considered intervention content, delivery and context when reviewing intervention effectiveness, as per current research guidance [51]. We analysed intervention content using both the PAPA and the BCT Taxonomy $[18,20]$. This two-tiered analysis approach encompasses both outcome and process, as recommended in the Medical Research Council guidance for developing and evaluating complex interventions [51]. With regards to delivery, we looked at pharmacist training, delivery channels (pharmacist-led or collaborative care), and delivery modes (e.g. face-to-face individual sessions). In terms of context, we went beyond looking at study location (country) and explored national healthcare policies, pharmacy guidelines, and pharmacy literature to explain the differences seen in intervention effectiveness.

The review is limited by the small number of included studies, suggesting a need for further research in this area. The generalisability of our findings is limited because we were unable to conduct moderator and publication bias analyses. The reliability of our findings is limited by the moderate to high risk of bias across the review. However, behavioural intervention trials often cannot meet the criteria in the Cochrane Collaboration's risk of bias tool because it was developed based on clinical RCTs [17].

We did not analyse data for asthma control, the most common primary outcome. However, it was felt that a review of data on adherence to asthma medication and subsequent asthma control was covered in a recent Cochrane review, so we did not duplicate this [44]. The guidelines for a small, medium and large effect size $(d)$ do not translate directly into the healthcare realm. A small effect size for one condition may have a larger clinical impact than a large effect size for another condition [52].

\section{Implications for clinical practice and future research}

In clinical practice, pharmacists can act as "medication experts" providing updated information and ongoing support for people with long-term conditions. Community pharmacy interventions such as the New Medicine Service can significantly increase adherence [53]. These interventions offer convenient access points to the healthcare system and ensure consistent contact with people through the provision of ongoing pharmaceutical care.

Our findings suggest that pharmacist-led interventions may be more effective if they are integrated into government policy and existing healthcare services. Notably, NHS England recently extended the Clinical Pharmacists in General Practice pilot [10]. These embedded pharmacists are working to improve patient safety and quality of care, whilst reducing the pressure on general practitioners.

Further RCTs investigating the effect of pharmacist-led interventions on adherence in asthma are needed. However, this review has provided an important summary of evidence to date highlighting the potential of pharmacist-led interventions. Future studies should aim to reduce the risk of detection bias and contamination between groups. Electronic monitoring devices should be used to measure adherence, although objective data (e.g. prescription refill rates) combined with blinded outcome assessors may be more pragmatic for pharmacy-based research. Adequately powered cluster-randomised trials will help reduce the risk of contamination between groups.

To build a reliable evidence base for effective interventions, future publications should report intervention content in sufficient detail. This may involve publishing an intervention protocol separately. Intervention fidelity measures are needed to ensure that interventions are being delivered as reported. Most included studies were conducted in Western cultures [23-29,33] and the cross-cultural applicability of our findings should be explored. Investigating the clinical relevance of effect estimates $(e . g . d)$ will help frame the real-life impact of research findings for people with asthma.

As outlined by the Lancet Asthma Commission, monitoring adherence in asthma will be an iterative and adaptive process requiring multidisciplinary input [7]. This review, combined with existing evidence, suggests that pharmacist-led interventions can effectively contribute to that process.

Author contributions: M.A. Mes: study design, literature search, data collection, data analysis, data interpretation and writing. C.B. Katzer: literature search, data analysis and data interpretation. A.H.Y. Chan: data analysis, data interpretation and writing. V. Wileman: data analysis, data interpretation and writing. S.J.C. Taylor: study design, literature search, data interpretation and writing. R. Horne: study design, literature search, data interpretation and writing.

Conflict of interest: M.A. Mes reports grants from the National Institute for Health Research Collaboration for Leadership in Applied Health Research and Care (NIHR CLAHRC) North Thames, during the conduct of the study; personal fees for consultancy on two previous projects from Spoonful of Sugar Limited, a UCL-spin-out behaviour change consultancy company, outside the submitted work; and is undertaking a $\mathrm{PhD}$ project that is affiliated with the Asthma UK Centre for Applied Research (AUKCAR), who covered attendance fees for AUKCAR Annual Scientific Meetings and AUKCAR PPI Fairs during the study period. C.B. Katzer reports grants from NIHR CLAHRC North 
Thames, during the conduct of the study; personal fees for consultancy on two previous projects from Spoonful of Sugar Limited, a UCL-spin-out behaviour change consultancy company, outside the submitted work; and is undertaking a PhD project is affiliated with the AUKCAR, who covered attendance fees for AUKCAR Annual Scientific Meetings and AUKCAR PPI Fairs during the study period. A.H.Y. Chan reports educational grants and consultancy fees from Janssen-Cilag, speaker fees from Novartis, consultancy fees from Spoonful of Sugar Ltd and a research award from Medicines New Zealand, outside the submitted work. V. Wileman reports grants from NIHR CLAHRC North Thames, during the conduct of the study. R. Horne reports grants from NIHR CLAHRC North Thames, during the conduct of the study; and has received grants from the AUKCAR; is a member of the academic arm of Medical Innovation Academic Consortium (CASMI); has received fees for speaker engagements from AbbVie, Amgen, Biogen, Idec, Gilead Sciences, GlaxoSmithKline, Janssen, Pfizer, Roche, Shire Pharmaceuticals, MSD, Astellas, AstraZeneca, DRSU, Novartis, Universitatsklinikum Hamburg-Eppendorf and Teva Pharmaceuticals; and is founder of UCL business spin-out company Spoonful of Sugar Ltd, outside the submitted work.

Support statement: This research was funded by the National Institute for Health Research Collaboration for Leadership in Applied Health Research and Care North Thames at Bart's Health NHS Trust (NIHR CLAHRC North Thames). The views expressed in this article are those of the authors and not necessarily those of the NHS, the NIHR or the Department of Health and Social Care. The NIHR CLAHRC North Thames was not involved in the writing of the manuscript or decision to submit for publication. No payment from a pharmaceutical company or other agency was received to write this article. Funding information for this article has been deposited with the Crossref Funder Registry.

\section{References}

1 Global Burden of Disease Study 2013 Collaborators. Global, regional, and national incidence, prevalence, and years lived with disability for 301 acute and chronic diseases and injuries in 188 countries, 1990-2013: a systematic analysis for the Global Burden of Disease Study 2013. Lancet 2015; 386: 743-800.

2 Masoli M, Fabian D, Holt S, et al. The global burden of asthma: executive summary of the GINA Dissemination Committee report. Allergy 2004; 59: 469-478.

3 The Global Asthma Network. The Global Asthma Report 2014. Auckland, New Zealand, The Global Asthma Network, 2014.

4 Mukherjee M, Stoddart A, Gupta RP, et al. The epidemiology, healthcare and societal burden and costs of asthma in the UK and its member nations: analyses of standalone and linked national databases. BMC Med 2016; 14: 113.

5 Pawankar R. Allergic diseases and asthma: a global public health concern and a call to action. World Allergy Organ J 2014; 7: 12 .

6 Global Burden of Disease Study 2015 Chronic Respiratory Collaborators. Global, regional, and national deaths, prevalence, disability-adjusted life years, and years lived with disability for chronic obstructive pulmonary disease and asthma, 1990-2015: a systematic analysis for the Global Burden of Disease Study 2015. Lancet Respir Med 2017; 5: 691-706.

7 Pavord ID, Beasley R, Agusti A, et al. After asthma: redefining airways diseases. Lancet 2018; 391: 350-400.

8 Murphy AC, Proeschal A, Brightling CE, et al. The relationship between clinical outcomes and medication adherence in difficult-to-control asthma. Thorax 2012; 67: 751-753.

9 Boyd MJ, Elliott RA, Barber N, et al. The impact of the New Medicines Service (NMS) in England on patients adherence to their medicines. Int J Pharm Pract 2014; 22: 66.

10 NHS England. General Practice Forward View. Redditch, NHS England, 2016.

11 Latif A, Pollock K, Boardman HF. The contribution of the Medicines Use Review (MUR) consultation to counseling practice in community pharmacies. Patient Educ Couns 2011; 83: 336-344.

12 Department of Health and Social Care. Transforming primary care: safe, proactive, personalised care for those who need it most. London, Department of Health and Social Care, 2014.

13 Horne R, Clatworthy J. Adherence to advice and treatment. In: French D, Vedhara K, Keptein AA, Weinman J, eds. Health Psychology. Oxford, BPS Blackwell, 2010; pp. 175-188.

14 Moher D, Liberati A, Tetzlaff J, et al. Preferred reporting items for systematic reviews and meta-analyses: the PRISMA statement. PLoS Med 2009; 6: e1000097.

15 Higgins JP, Green S. Cochrane Handbook for Systematic Reviews of Interventions Version 5.1.0 [Updated March 2011]. London, The Cochrane Collaboration, 2011.

16 Lefebvre C, Eisinga A, McDonald S, et al. Enhancing access to reports of randomized trials published world-wide - the contribution of EMBASE records to the Cochrane Central Register of Controlled Trials (CENTRAL) in The Cochrane Library. Emerg Themes Epidemiol 2008; 5: 13.

17 Higgins JP, Altman DG, Gøtzsche PC, et al. The Cochrane Collaboration's tool for assessing risk of bias in randomised trials. BMJ 2011; 343: d5928.

18 Horne R. Compliance, adherence, and concordance: implications for asthma treatment. Chest 2006; 130: Suppl, $65 \mathrm{~s}-72 \mathrm{~s}$.

19 Nunes V, Neilson J, O'Flynn N, et al. Medicines adherence: involving patients in decisions about prescribed medicines and supporting adherence. London, UK, National Institute for Health and Care Excellence (NICE); 2009.

20 Michie S, Richardson M, Johnston M, et al. The Behavior Change Technique Taxonomy (v1) of 93 Hierarchically Clustered Techniques: Building an International Consensus for the Reporting of Behavior Change Interventions. Ann Behav Med 2013; 46: 81-95.

21 Health Services Research Unit. Database of ICCs. Aberdeen, University of Aberdeen, 2004.

22 Campbell M, Grimshaw J, Steen N. Sample size calculations for cluster randomised trials. J Health Serv Res Policy 2000; 5: 12-16.

23 Armour C, Bosnic-Anticevich S, Brillant M, et al. Pharmacy Asthma Care Program (PACP) improves outcomes for patients in the community. Thorax 2007; 62: 496-502.

24 Charrois TL, Newman SC, Senthilselvan A, et al. Improving asthma control in the rural setting: The BREATHE (Better Respiratory Education and Asthma Treatment in Hinton and Edson) study. Can Pharm J 2006; 139: $44-50$. 
25 Cordina M, McElnay JC, Hughes CM. Assessment of a community pharmacy-based program for patients with asthma. Pharmacotherapy 2001; 21: 1196-1203.

26 García-Cárdenas V, Sabater-Hernández D, Kenny P, et al. Effect of a pharmacist intervention on asthma control. A cluster randomised trial. Respir Med 2013; 107: 1346-1355.

27 Manfrin A, Tinelli M, Thomas T, et al. A cluster randomised control trial to evaluate the effectiveness and cost-effectiveness of the Italian medicines use review (I-MUR) for asthma patients. BMC Health Serv Res 2017; 17: 300.

28 Mehuys E, van Bortel L, Bolle L, et al. Effectiveness of pharmacist intervention for asthma control improvement. Eur Respir J 2008; 31: 790-799.

29 Munzenberger PJ, Hill MJ. Impact of an asthma-specific questionnaire on problem identification and clinical and economic outcomes in ambulatory patients with persistent asthma. J Am Pharm Assoc 2007; 47: 147-155.

30 Wang KY, Chian CF, Lai HR, et al. Clinical pharmacist counseling improves outcomes for Taiwanese asthma patients. Pharm World Sci 2010; 32: 721-729.

31 Wong LY, Chua SS, Husin AR, et al. A pharmacy management service for adults with asthma: a cluster randomised controlled trial. Fam Pract 2017; 34: 564-573.

32 Xaubet Olivera CM, Vianna EO, Bonizio RC, et al. Asthma self-management model: randomized controlled trial. Health Educ Res 2016; 31: 639-652.

33 Young HN, Havican SN, Griesbach S, et al. Patient and phaRmacist telephonic encounters (PARTE) in an underserved rural patient population with asthma: results of a pilot study. Telemed J E Health 2012; 18: 427-433.

34 Cohen J. Statistical power analysis for the behavioral sciences. 2nd Edn. Hillsdale, NJ, Lawrence Earlbaum Associates, 1988.

35 Horne R. Compliance, adherence and concordance. In: Taylor K, Harding G, eds. Pharm Pract (Granada). London, UK, Taylor and Francis, 2001; pp. 165-184.

36 Kelly MP, Barker M. Why is changing health-related behaviour so difficult? Public Health 2016; 136: 109-116.

37 Marteau TM, Hollands GJ, Kelly MP. Changing population behaviour and reducing health disparities: exploring the potential of 'choice architecture' interventions. Rockville, Agency for Healthcare Research and Quality, 2015. http://www.ahrq.gov/professionals/education/curriculum-tools/population-health/marteau.html.

38 Benrimoj SI, Frommer MS. Community pharmacy in Australia. Aust Health Rev 2004; 28: 238-246.

39 Elsen C, Libert M. Health promotion in community pharmacy - The situation in Belgium. Brussels, The European Commission, 2001.

40 Gastelurrutia MA, Faus MJ, Fernández-Llimós F. Providing patient care in community pharmacies in Spain. Ann Pharmacother 2005; 39: 2105-2110.

41 Eurostat. Healthcare personnel statistics - dentists, pharmacists and physiotherapists. European Union, 2017.

42 Karim Z, Noott A. An overview of pharmacy practice in Canada. Tomorrow's Pharmacist 2011.

43 Christensen DB, Farris KB. Pharmaceutical care in community pharmacies: practice and research in the US. Ann Pharmacother 2006; 40: 1400-1406.

44 Normansell R, Kew KM, Stovold E. Interventions to improve adherence to inhaled steroids for asthma. Cochrane Database Syst Rev 2017; 4: CD012226.

45 Hughes CM, Hawwa AF, Scullin C, et al. Provision of pharmaceutical care by community pharmacists: a comparison across Europe. Pharm World Sci 2010; 32: 472-487.

46 Preference Collaborative Review Group. Patients' preferences within randomised trials: systematic review and patient level meta-analysis. BMJ 2009; 338: 85-88.

47 Lam WY, Fresco P. Medication Adherence Measures: An Overview. BioMed Res Int 2015; 2015: 12.

48 McCambridge J, Kypri K, Elbourne D. In randomization we trust? There are overlooked problems in experimenting with people in behavioral intervention trials. J Clin Epidemiol 2014; 67: 247-253.

49 Sangeda RZ, Mosha F, Prosperi M, et al. Pharmacy refill adherence outperforms self-reported methods in predicting HIV therapy outcome in resource-limited settings. BMC Public Health 2014; 14: 1035.

50 AL-Jahdali $\mathrm{H}$, Ahmed A, AL-Harbi A, et al. Improper inhaler technique is associated with poor asthma control and frequent emergency department visits. Allergy Asthma Clin Immunol 2013; 9: 8

51 Craig P, Dieppe P, Macintyre S, et al. Developing and evaluating complex interventions: the new Medical Research Council guidance. $B M J$ 2008; 337: a1655.

52 McGough JJ, Faraone SV. Estimating the size of treatment effects: moving beyond p values. Psychiatry (Edgmont) 2009; 6: 21-29.

53 Elliott RA, Boyd MJ, Salema NE, et al. Supporting adherence for people starting a new medication for a long-term condition through community pharmacies: A pragmatic randomised controlled trial of the New Medicine Service. BMJ Qual Safety 2016; 25: 747-758. 